\title{
FÍSICA CONTEMPORÁNEA EN LA ESCUELA SECUNDARIA: UNA EXPERIENCIA EN EL AULA INVOLUCRANDO FORMACIÓN DE PROFESORES
}

\author{
OSTERMANN, FERNANDA y MOREIRA, MARCO ANTONIO \\ Instituto de Física. Universidade Federal do Rio Grande do Sul \\ CP 15051, 91501-970, Porto Alegre, RS Brasil \\ E-mail: fernanda@if.ufrgs.br - moreira@if.ufrgs.br
}

\begin{abstract}
SUMMARY
In this paper we describe our experience in teaching two contemporary physics topics -elementary particles and superconductivity- at high school level. The experience was developed in two years, including topic selection, preparation of instructional materials, teacher training, and classroom implementation.
\end{abstract}

\section{INTRODUCCIÓN}

En los Estados Unidos y, probablemente, en un nivel internacional, la preocupación por la enseñanza de la física moderna y contemporánea (FMC) en las escuelas y en los cursos básicos de la universidad ha empezado o se ha intensificado con la «Conferencia sobre la Enseñanza de la física moderna», realizada en el Fermilab, en Batavia (Illinois), en abril de 1986, en la cual cerca de cien profesores interactuaron con una decena o más de físicos norteamericanos. El objetivo de la conferencia era el de promover la enseñanza de tópicos de investigación en física, en particular física de partículas y cosmología, en las escuelas secundarias y en las asignaturas de física general en las universidades (Aubrecht, 1986). Sin embargo, son muchas las razones presentadas en la literatura respecto a la necesidad de actualizar los currículos de física, en particular en secundaria (Gil et al., 1987; Barojas, 1988; Aubrecht, 1989; Stannard, 1990; Kalmus, 1992; Wilson, 1992; Swinbank, 1992; Terrazzan, 1992 y 1994). Entre dichas razones se destacan:
- Despertar la curiosidad de los alumnos y ayudarlos a reconocer la física como una empresa humana y, por lo tanto, cercana a ellos.

- Los estudiantes no tienen contacto con el excitante mundo de la física actual, pues la física que ven no pasa de 1900. Dicha situación es inaceptable en un siglo en el cual ideas revolucionarias han cambiado totalmente la ciencia.

- Es necesario motivar a los jóvenes para la carrera científica. Son ellos los futuros profesores e investigadores en física. La física moderna y contemporánea es la que más puede influenciar a los estudiantes a elegir física como carrera profesional.

- Los estudiantes oyen hablar de temas como agujeros negros y big bang en la televisión o en películas de ficción científica, pero jamás en clases de física. 
- La física moderna y contemporánea es considerada difícil y abstracta; no obstante, las investigaciones en enseñanza de la física han mostrado que la física clásica también es difícil y abstracta para los alumnos, que presentan serias dificultades conceptuales para comprenderla.

- La enseñanza de temas actuales de la física puede contribuir para transmitir a los alumnos una visión más correcta de esa ciencia y de la naturaleza del trabajo científico, superando la visión lineal, netamente acumulativa del desarrollo científico que impregna los libros de texto y las clases de física hoy utilizados.

Por otro lado, a pesar de todos esos argumentos, no hay mucho en la literatura con respecto a cómo enfocar los temas modernos y contemporáneos en la escuela secundaria. De una manera general, son tres las vertientes más representativas de propuestas de enfoques metodológicos para introducir la física moderna y contemporánea en la enseñanza media: la exploración de los límites de los modelos clásicos; la no-utilización de referencias a los modelos clásicos; la elección de tópicos esenciales (Terrazzan, 1994; Camargo, 1996; Pereira, 1997; Alvetti y Delizoicov, 1998).

La primera vertiente -exploración de los límites clásicos- se debe a los trabajos de Gil y Solbes, investigadores de la Universidad de Valencia. En uno de sus trabajos (Gil et al., 1988), estos autores muestran, a partir del análisis de cuarenta y dos libros didácticos de física usados en España, que la mayoría de éstos no hacen ninguna referencia: $a$ ) al carácter no lineal del desarrollo científico; $b$ ) a las dificultades que generaron las crisis de la física clásica; $c$ ) a las profundas diferencias conceptuales entre la física clásica y la moderna.

Para estos autores, esta visión simplista bajo la cual se enseña la física en las escuelas genera serias concepciones alternativas en los alumnos. A través de una encuesta hecha con 536 alumnos, con edades comprendidas entre 16 y 18 años, estos investigadores han verificado que la gran mayoría ignoraba la existencia de una crisis en el desarrollo de la física clásica, desconocía la diferencia entre física clásica y moderna, y presentaba concepciones erróneas respecto a la dualidad onda-partícula, a la ecuación de Einstein $\left(E=m c^{2}\right)$ y al concepto de partícula elemental. Basándose en eso, sugieren, entonces, que los conceptos de física moderna y contemporánea sean introducidos teniendo como referente un modelo constructivista de enseñanza-aprendizaje en la perspectiva de un cambio conceptual y metodológico (Gil et al., 1987; Solbes et al., 1987a; Gil y Solbes, 1993).

La segunda vertiente -la no-utilización de referencias a los modelos clásicos- se atribuye a las investigaciones de Fischler y Lichtfeldt (1992) de la Universidad Libre de Berlín. En buena medida en oposición a la vertiente anterior, estos investigadores consideran que el aprendizaje de la física moderna y contemporánea es dificultado por el uso frecuente de analogías clásicas. Por ejemplo, el átomo de Bohr, una vez aprendido, pasa a ser un obstáculo para el entendimiento de teorías más modernas.
La tercera vertiente -elección de tópicos esenciales- es contribución de Arons (1990), de la Universidad de Washington, que propone que sólo unos pocos conceptos de física moderna y contemporánea deben ser enseñados en la escuela media. Según Arons, lo más importante en un curso introductorio de física moderna y contemporánea es proporcionar a los alumnos «alguna percepción» respecto a conceptos como electrones, fotones, núcleos y estructura atómica. Él defiende también la búsqueda de apoyo en la física clásica para abordar los tópicos modernos y contemporáneos. $\mathrm{O}$ sea, es necesario buscar en la física clásica los prerequisitos esenciales para que los nuevos tópicos sean comprensibles.

En resumen, lo que se puede percibir en la literatura con respecto a cómo introducir tópicos de física moderna y contemporánea en cursos introductorios de física es que hay divergencias en relación con el camino que se debe seguir y que son pocos los estudios en el área. En particular, el papel de las analogías clásicas, el énfasis o no en prerequisitos, el enfoque histórico o lógico son puntos en los que hay mucho desacuerdo. Como bien sugiere Stefanel (1998), este movimiento de reformulación curricular recién empieza y todavía son necesarias muchas investigaciones hasta que se sepa más acerca de esta compleja problemática.

Respecto a qué enseñar sobre temas modernos y contemporáneos tampoco es mucho lo que se encuentra en la literatura, pero se observa una concentración en los siguientes tópicos: relatividad, partículas elementales y mecánica cuántica (Solbes et al., 1987 a; Gil et al., 1988; Moreira, 1990; Galetti, 1990; Farmelo, 1992; Jones, 1992; Swinbank, 1992; Barlow, 1992; Ireson, 1996; Allday, 1997; Contemporary Physics Education Project, 1998; Fermilab, 1998). Sin embargo, la cuestión del contenido es la que está más discutida en la literatura sobre la enseñanza de la física moderna y contemporánea, comparada con aspectos metodológicos, epistemológicos, históricos, curriculares y propuestas evaluadas en el aula.

Hay también algunos estudios relativos a concepciones alternativas de los alumnos (Solbes et al., 1987b; Lijnse et al., 1990; De Posada et al., 1990; Alemañ Berenguer, 1997), en general asociadas a errores conceptuales de los libros de texto o a informaciones vehiculizadas por los medios de comunicación.

En lo que se refiere a propuestas llevadas al aula y evaluadas, los trabajos de Gil y otros (1987 y 1988), Solbes y otros (1987a), Fischler y Lichtfeldt (1992), Cuppari y otros (1997) y Stefanel (1998) sugieren la posibilidad de éxito en la inserción de temas más actuales en el currículo de las escuelas. No obstante, son necesarios más estudios de esa naturaleza para que se tenga un conocimiento más amplio en ese campo.

En resumen, en la literatura referida a física moderna y contemporánea en el currículo de física, en particular en secundaria, hay varios argumentos a favor de la inclusión de tópicos actuales en ese currículo, divergencias metodológicas respecto a cómo incluirlos, algunas suge- 
rencias relativas a qué tópicos incluir y algunas investigaciones sobre experiencias en el aula. Áreas importantes de la física moderna y contemporánea no están contempladas en dicha literatura. Asimismo, los temas de la formación de los profesores para enseñar esos tópicos y de la preparación de materiales instruccionales adecuados para profesores y alumnos también están poco trabajados en los artículos.

Partiendo de la premisa de que es importante (y urgente) actualizar el currículo de física en la escuela secundaria y de la constatación de que todavía hay mucho que hacer para alcanzar este objetivo, realizamos un estudio involucrando la identificación de tópicos de física moderna y contemporánea según la opinión de expertos, la elaboración de materiales instruccionales para la enseñanza de dos de esos tópicos, la preparación de profesores para enseñar esos dos tópicos y, finalmente, su utilización, en el aula, con estudiantes de secundaria. Dicha investigación, que fue realizada en Brasil, a lo largo de 1997 y 1998, se describe a continuación.

\section{LA IDENTIFICACIÓN DE LOS TÓPICOS A TRAVES DE UN ESTUDIO DELPHI}

Con el objetivo de llegar a una lista consensuada de tópicos de física contemporánea que deberían ser enseñados en la escuela media si quisiéramos actualizar el currículo de física en ese nivel, realizamos una encuesta con físicos, investigadores en enseñanza de la física y profesores de física, a través de la técnica Delphi (Linstone y Turoff, 1975). Esta técnica fue desarrollada por la compañía norteamericana Rand Corporation, en los años cincuenta, con el objetivo de resolver problemas que exigían opiniones de grupos de personas. Es una técnica de búsqueda de consenso por aproximaciones sucesivas. En nuestro caso, el estudio Delphi tuvo tres etapas y la participación inicial de 54 físicos, 22 investigadores en enseñanza de la física y 22 profesores de física (Ostermann y Moreira, 1998). En la primera etapa, a los sujetos, se les pidió solamente que propusieran tópicos de física contemporánea que en su opinión deberían ser contemplados para su inclusión en el currículo de física en secundaria si se quisiera actualizar. En la segunda etapa se les pidió una toma de posición, en términos de estar o no de acuerdo, con respecto a todos los tópicos propuestos en la primera etapa. Finalmente, en la tercera etapa se les dio la oportunidad de revisar su posición anterior frente al resultado (la tabulación) de la segunda etapa.

La lista final de tópicos resultante de este proceso ${ }^{1}$ (con un promedio arriba de 3,5 en un máximo de 5) fue la siguiente: efecto fotoeléctrico, átomo de Bohr, leyes de conservación, radioactividad, fuerzas fundamentales, dualidad onda-partícula, fisión y fusión nucleares, origen del universo, rayos $\mathrm{X}$, metales y aisladores, semiconductores, lásers, superconductores, partículas elementales, relatividad especial, big bang, estructura molecular, fibras ópticas. Ésta es la lista consensual de los expertos (físicos, investigadores en enseñanza de la física y profesores de física en secundaria) referida a los tópicos de física contemporánea que deberían ser enseñados en secundaria si fuera para actualizar el currículo de física en ese nivel. Como se ve, los participantes han sugerido muchos tópicos que encajarían mejor en la llamada física moderna. Por física contemporánea hemos definido, explícitamente, en los cuestionarios utilizados, los desarrollos de la física a partir de, aproximadamente, los años cuarenta. Sin embargo, muchos de los tópicos propuestos son de fines del siglo pasado y comienzos de éste. Considerando que lo que se enseña de física en secundaria en general no llega a este siglo, quizás no esté mal hablar de física moderna y contemporánea en vez de física contemporánea.

Una vez obtenida la lista de tópicos, nuestro problema pasó a ser la elección de uno o dos de ellos para trabajar con los alumnos en el aula. Considerando que el foco de nuestro trabajo era la enseñanza de tópicos de física contemporánea, decidimos no tener necesariamente en cuenta algunos de esos tópicos, aunque estuviesen entre los primeros de la lista. Por otro lado, no teníamos ningún compromiso a prioricon los primeros de la «lista de los expertos» que encajasen en ese foco. Es decir, el estudio Delphi fue hecho para llegar a una lista consensual de tópicos con el fin de orientar nuestro trabajo, no para que tuviéramos que seguirla paso a paso. Utilizamos, entonces, nuestros propios criterios en la elección de tópicos. Dichos criterios han involucrado aspectos didáctico-pedagógicos y epistemológicos, así como el dominio del contenido específico. Creemos que para actualizar el currículo de física en las escuelas es imprescindible un conocimiento riguroso de la física involucrada para que sea adecuada la transposición didáctica de los temas. Sin un buen dominio de los contenidos contemporáneos involucrados, la elaboración de materiales didácticos y la preparación de profesores puede quedar comprometida. Los tópicos elegidos fueron superconductividad y partículas elementales y la justificación fue la que se expone a continuación.

\section{JUSTIFICACIÓN DE LA ELECCIÓN DE SUPERCONDUCTIVIDAD Y PARTÍCULAS ELEMENTALES}

En primer lugar, elegimos esos tópicos porque encajan bien en la llamada física contemporánea (la física desde la década de los años cuarenta hasta hoy). Sin embargo, se puede justificar cada uno de ellos en términos didácticos.

El fenómeno de la superconductividad encaja bien en un importante énfasis curricular, el de las relaciones entre ciencia, tecnología y sociedad (CTS). Este énfasis considera fundamental que los alumnos perciban los conocimientos científicos en el contexto de sus aplicaciones tecnológicas y los utilicen en pleno ejercicio de la ciudadanía. La superconductividad está relacionada con la impresionante revolución tecnológica que estamos viviendo en este final de siglo, ilustrando así una serie de aplicaciones potencialmente motivadoras para los alum- 
nos. Además de eso, la comprensión de este tópico requiere contenidos de física clásica (por ejemplo, termodinámica y electromagnetismo) normalmente estudiados en secundaria así como conceptos básicos de mecánica cuántica y estado sólido. Eso significa que el tema de la superconductividad puede ser incorporado al currículo de manera bien articulada tendiendo un puente entre contenidos clásicos y modernos. Finalmente, hay que decir que dicho tema permite alguna actividad experimental, rechazando la crítica de que los contenidos contemporáneos son muy teóricos. En particular, se puede demostrar el fenómeno de la levitación usando una pastilla superconductora, un pequeño imán y un poco de nitrógeno líquido, lo que permite a los alumnos «ver» el fenómeno.

El tema de las partículas elementales, a su vez, está vinculado a cuestiones más básicas de la física y puede remitirnos, quizás, a problemas filosóficos (como el de buscar orden en la diversidad) que la superconductividad, con su carácter más técnico, no presenta. La pregunta clave de este campo de la física - ¿cómo funciona el universo?- es una pregunta que siempre ha fascinado al hombre, tanto desde el punto de vista microscópico como del macroscópico. Por ende, el tema es potencialmente motivador. El estudio de las partículas elementales es muy adecuado para ilustrar la interacción entre el pensar y el hacer en la producción del conocimiento científico. Por ejemplo, varias predicciones teóricas con respecto a la existencia de algunas partículas anticiparon los resultados de la experimentación, confirmando la creencia epistemológica contemporánea de la inseparabilidad entre presupuestos teóricos y experimentación. La idea de modelización y búsqueda de simetría es otra característica de la física que el tópico partículas elementales ilustra muy bien. En una cierta época lo que se tenía era un catálogo de partículas, pero la búsqueda obstinada de simetrías en la naturaleza, la creencia de que ese catálogo podría ser explicado a través de un modelo llevó al «modelo estándar». Con el tema partículas se puede fácilmente presentar la física como una ciencia viva, dinámica. El «modelo estándar» es el modelo que se tiene hoy para este campo de la física, pero, obviamente, puede cambiar. Sin enfatizar la memorización de nombres y clasificaciones se puede facilitar la construcción por parte del alumno de la idea de la física de partículas como una empresa humana que busca organizar la comprensión del mundo a través de los quarks y leptones y de las cuatro interacciones fundamentales. Es notorio el esfuerzo internacional que se está haciendo para introducir este tema en niveles introductorios de escolarización. No es difícil percibir la potencialidad del tema, tanto para una relectura de la física clásica (en sus leyes de conservación, por ejemplo) como para una visión cosmológica actualizada, que permita reconocer la física como un cuerpo de conocimientos altamente estructurado, no como una obra de retazos.

Este tópico (así como muchos otros contemporáneos) requiere el uso de analogías para la comprensión de algunos de sus aspectos. Por ejemplo, las interacciones, desde el punto de vista de la física moderna y contempo- ránea son entendidas a través del intercambio de una partícula mediadora. Propusimos, entonces, a los alumnos que intentasen captar el significado de esta idea a través de una analogía con el juego del bumerán, en el cual dos jugadores (de espaldas) interactúan (se atraen) a través del intercambio del bumerán. Sin duda, un puente que se puede tender para introducir el tema de las partículas elementales son los modelos atómicos enseñados en las clases de química. Al proporcionar una visión contemporánea de átomo, estaremos intentando romper con el modelo planetario tan frecuentemente presentado en esas clases.

A pesar de aparentemente denso, lleno de nombres y clasificaciones, el tópico «partículas» culmina en una síntesis que organiza de forma extraordinaria la comprensión del universo. Creemos, entonces, que a través de tópicos como éste se puede ayudar a los alumnos a percibir que es posible estructurar el conocimiento físico de manera organizada y resumida, utilizando, por ejemplo, unas pocas leyes de conservación. La idea de que son sólo cuatro las interacciones fundamentales también puede ayudar a los alumnos a reconocer la unificación como un valor importante para la física. Naturalmente es necesario ayudarlos a hacer la reconciliación integradora que les permita encajar en sólo cuatro tipos todas las fuerzas que conocen.

Otra y no menos importante razón para elegir esos tópicos es que teníamos el conocimiento físico adecuado para preparar materiales y profesores en los dos. Incluso, la primera autora de este trabajo ha participado en un curso de un mes, en el Fermilab, sobre la enseñanza de partículas elementales. Reiteramos que, desde nuestro punto de vista, el dominio de los contenidos parece ser una condición previa para intentar cualquier acción en el sentido de trabajar esos contenidos en la escuela media. Recíprocamente, las dificultades de los profesores con los contenidos contemporáneos parecen ser un obstáculo para su inclusión en el currículo.

\section{METODOLOGÍA}

Nuestro diseño no es experimental. No hicimos elección al azar ni de sujetos ni de escuelas. Trabajamos con todos los estudiantes que teníamos matriculados en la asignatura «Práctica de enseñanza de la física» y con todos los alumnos que tenían clases de física en las escuelas que aceptaron recibir a nuestros estudiantes para su práctica supervisionada. Tampoco consideramos que nuestros estudiantes o las escuelas en las cuales trabajamos son casos estudiados. Creemos que nuestro estudio es más bien descriptivo: nos interesa hacer una descripción con esos estudiantes, alumnos y escuelas, dentro de las condiciones que teníamos, para introducir un par de tópicos de física contemporánea en el currículo y qué ha pasado en consecuencia. Trabajamos con futuros profesores de física y alumnos de secundaria en física tal como los teníamos a mano e hicimos una intervención didáctica que suponemos innovadora y que vamos a describir sin ninguna pretensión de generalización ni de 
excesiva profundización. En el relato de la preparación de los materiales y de los profesores, así como de la enseñanza de los tópicos en las escuelas, quedará más clara la metodología de nuestro trabajo.

\section{MARCO TEÓRICO}

Nuestro marco teórico es el del aprendizaje significativo receptivo de Ausubel y Novak (Ausubel, 1963, 1968; Ausubel y Novak, 1983; Novak y Gowin, 1988) según el cual las condiciones para el aprendizaje significativo son que el alumno presente una predisposición para aprender y que los materiales educativos sean potencialmente significativos. Esta segunda condición implica que los materiales tengan significado lógico y que el alumno tenga en su estructura cognoscitiva los «subsunsores» específicamente relevantes o, en otras palabras, el conocimiento previo adecuado. Una vez satisfechas esas dos condiciones, el aprendizaje puede ser receptivo en el sentido de que la nueva información se presenta al alumno en su forma final, no hay que descubrirla sino procesarla. Por más creativa que sea la manera de presentar el nuevo conocimiento, se trata de aprendizaje receptivo, lo que no significa pasivo ni transmisivo.

En el aprendizaje significativo receptivo, que según Ausubel es el mecanismo humano por excelencia para dar significado a los nuevos conocimientos, recepción no significa pasividad, pues el sujeto que aprende debe hacer un esfuerzo deliberado para relacionar el nuevo material con su estructura cognoscitiva de manera sustantiva y no arbitraria. Tampoco significa transmisividad, pues el aprendizaje significativo es idiosincrático y nadie puede transmitir nada a nadie, nadie puede aprender. Es un error confundir aprendizaje receptivo con aprendizaje pasivo, mecánico, no constructivista. El enfoque de Ausubel y Novak considera al alumno como constructor de su propio conocimiento y como persona. Sin embargo, es una persona que para hacer su construcción, típicamente, recibe la información, capta su significado y, si quiere aprender de manera significativa, presenta una disposición para relacionarla de manera no literal y no arbitraria con su conocimiento previo. Por supuesto que este enfoque no dispensa buenas clases, buenos materiales, mucha interacción personal, actividades prácticas, recursos motivacionales, resolución de problemas, evaluaciones coherentes con los procedimientos didácticos y otras características de una buena enseñanza. La diferencia es que el profesor no debe sentirse culpado o avergonzado por estimular el aprendizaje receptivo, pues es ése nuestro mecanismo típico de aprendizaje.

\section{LA PREPARACIÓN DE LOS MATERIALES Y DE LOS PROFESORES}

Hecha la elección de los tópicos, pasamos a ocuparnos de la elaboración de materiales educativos y, simultáneamente, de la preparación de los profesores. Nos pareció que la inserción de esos tópicos en la escuela secundaria dependería de esos dos factores, los materiales y los profesores. En realidad, la actualización del currículo de física en secundaria, a través de cualesquiera tópicos, depende crucialmente de materiales de buena calidad y de profesores bien preparados y motivados para el cambio. Al inicio, más preocupada con el alumno (sus preconcepciones y la búsqueda del cambio conceptual), la investigación en enseñanza de las ciencias parece hoy inclinada a atacar el problema de la calidad de la enseñanza por la vía del profesor (Duit, 1993; Porlán y Rivero, 1998; Furió, 1994). Aparentemente, hay un consenso en que una mejor actuación de los profesores puede reflejar en el aprendizaje de los alumnos, contribuyendo a aumentar la calidad del proceso como un todo. La reestructuración de la enseñanza de la física en la escuela secundaria implica, por lo tanto, la incorporación de los resultados de la investigación en didáctica de la física a la formación de los profesores. Esos resultados ponen, por ejemplo, la necesidad de búsqueda de marcos teóricos para la práctica docente y cómo ésos pueden explicitar muchas metodologías empleadas en el aula.

Para el tópico partículas elementales preparamos un texto (Ostermann, 1998) de 54 páginas, para profesores, basado en varias referencias bibliográficas (Close, 1983; Schwarz, 1992; Williams, 1992; Physics Education, 27(2), 1992; Particle Physics Group, 1997; Fermilab, 1998). Este texto incluye una breve historia del desarrollo del concepto de átomo, los descubrimientos del inicio del siglo xx, las leyes de conservación, las interacciones fundamentales y el modelo estándar, además de ejercicios y sugerencias de actividades. Complementando el texto, preparamos diapositivas, transparencias y una tabla en formato póster, sobre quarks, leptones, partículas mediadoras e interacciones fundamentales.

Respecto al tópico superconductividad hicimos un trabajo semejante. Preparamos un texto de 74 páginas (Ostermann et al., 1998a, 1998b) para profesores de enseñanza media basado en varias referencias bibliográficas (Rose-Innes y Rhoderick, 1988; Cyrot y Pavuna, 1992; Luiz, 1992; Nobel, 1996; Pureur Neto, 1996). El texto incluye una breve descripción histórica del fenómeno, las propiedades básicas de un superconductor (resistividad nula y efecto Meissner), la termodinámica de la transición superconductora, la teoría de London, la teoría Ginzburg-Landau, la teoría BCS, la levitación magnética, los superconductores tipo II y las aplicaciones de la superconductividad. En el laboratorio produjimos una pastilla superconductora $\left(Y B a C u O, T_{\mathrm{c}}=92 \mathrm{~K}\right)$, del tamaño de una moneda, para fines demostrativos.

Los profesores que preparamos fueron 12 estudiantes del curso de formación de profesores de física de la Universidad Federal de Río Grande del Sur, en Porto Alegre (Brasil). Este curso es de cuatro años y está diseñado específicamente para la formación preservicio de profesores de física. Además de asignaturas teóricas y prácticas de física y matemáticas, hay unas cuantas asignaturas volcadas hacia la enseñanza de la física. Una de ésas, de último semestre, llamada «Práctica de ense- 
ñanza de la física» (EDU 02220 - 6 horas semanales), se caracteriza por proporcionar al futuro profesor una práctica docente supervisada en una escuela regular del sistema educativo público o privado. La estructura de esa asignatura es básicamente la siguiente: hay una parte práctica en la escuela (tres horas semanales) y una parte teórica desarrollada en la universidad (tres horas semanales). La primera parte es desarrollada a lo largo de tres meses: al inicio, los profesores-docentes observan y ayudan (a lo largo de dos meses) al profesor de física de una clase previamente definida y a posteriori dictan clases (a lo largo de un mes). El período de observación en la escuela involucra la familiarización del futuro docente en su contexto de práctica supervisionada. A través de la utilización de un «diario de a bordo», el futuro profesor puede trazar un perfil de la escuela, de la clase y del profesor. En ese período es necesario que asista a las clases del profesor asignado con sus alumnos, así como que recoja todos los datos que juzgue pertinentes para un buen conocimiento de la realidad escolar en la cual está inserto. Gradualmente puede empezar a ayudar al profesor en las clases de problemas o laboratorio, a través de un período de ayudantía. Paralelamente, los futuros docentes tienen clases teóricas en la universidad para que puedan dominar el contenido que va a ser enseñado, adecuando y organizando sus conocimientos para el trabajo en el aula. En el período llamado de regencia, los futuros docentes dictan un promedio de 10 horas de aula. Al final de la práctica supervisionada, los docentes-estudiantes hacen un informe (a lo largo de un mes) en el cual son descritas las etapas de su práctica. Todas esas etapas de la asignatura se desarrollan bajo la supervisión del profesor de «Práctica de enseñanza» (en nuestro caso, la primera autora), lo que incluye la observación de la mayoría de las clases de cada futuro profesor. El profesor de física de cada clase participa activamente del proceso, tanto para orientar cuestiones más prácticas de clase como también para compartir las innovaciones propuestas, pudiendo integrarlas posteriormente a su práctica docente también.

El grupo de 12 estudiantes fue dividido en dos de 6, uno encargado del tema de la superconductividad y el otro del tópico partículas elementales. En la parte de la asignatura «Práctica de Enseñanza», que correspondía a clases en la universidad, cada grupo tuvo clases teóricas y prácticas referidas a su tema (superconductividad o partículas). En dichas clases se utilizó todo el material instruccional ya preparado. Sin embargo, los futuros docentes tuvieron a su cargo la tarea de adaptarlos para uso en la escuela secundaria. Por ejemplo, a partir del texto del profesor que recibieron, tuvieron que elaborar el texto del alumno para usar en sus clases como material de apoyo.

\section{LA ENSEÑANZA DE LOS TÓPICOS EN LAS ESCUELAS}

\section{Partículas elementales}

El tópico partículas elementales fue enseñado en tres escuelas secundarias de Porto Alegre, dos públicas y una privada, para tres grupos de noveno grado (14-15 años), dos de décimo grado (15-16 años) y uno de décimo primero (16-17 años), que es el último de la escuela secundaria en Brasil. En total, fueron 126 alumnos y seis profesores. Las clases fueron dictadas en la perspectiva del aprendizaje receptivo, ilustradas con audiovisuales y ejercicios para los alumnos. Durante un mes, los futuros docentes elaboraron un texto («texto del alumno») bajo la supervisión del profesor de «Práctica», que procuraba simplificar y, de cierta manera, organizar el contenido para el nivel del alumno de secundaria. Ese texto de, aproximadamente, 20 hojas, tenía tres partes: modelos atómicos (pudín de pasas hasta el modelo actual), partículas elementales (quarks y leptones; hadrones) y las cuatro interacciones fundamentales. Una actividad que resultó interesante para los alumnos fue la de ilustrar un experimento (por ejemplo, el de Rutherford) de medidas indirectas en física de partículas. La actividad simula un experimento en el que el material blanco es bombardeado por partículas de alta velocidad y las colisiones son estudiadas. Con una hoja que contenga círculos en un área rectangular es posible, a través de colisiones de bolitas de vidrio aleatoriamente distribuidas sobre esa área, determinar el radio de los círculos.

\section{Superconductividad}

El tema de la superconductividad fue trabajado en dos escuelas públicas, con dos grupos de noveno grado, uno de décimo y tres de décimo primero, involucrando 145 alumnos y 6 profesores. Las clases fueron expositivas, con audiovisuales, demostración de la levitación y ejercicios para los estudiantes. Los futuros docentes prepararon un texto («texto del alumno») que intentaba simplificar y adaptar el contenido para el nivel del alumno de secundaria. El texto, que resultó de 21 hojas, tiene siete partes: revisión de la física clásica (modelo de metal, corriente, resistividad, ley de Faraday-Lenz), propiedades básicas de un superconductor (diferencia entre superconductor y conductor perfecto), cambio de estado, levitación magnética, teoría BCS (con analogías para su comprensión), superconductores de tipo II y aplicaciones tecnológicas. El «texto del alumno» tiene una parte introductoria hecha para revisar algunos aspectos de los conocimientos previos requeridos para el aprendizaje significativo de las propiedades básicas de los superconductores: un modelo de metal, corriente electrica, ley de Ampère y ley de Faraday-Lenz. La idea que está detrás del énfasis en la diferencia entre «superconductor y conductor perfecto» que aparece en el texto se expone para evidenciar sus distintas respuestas magnéticas: los superconductores reaccionan a campos magneticos estáticos (efecto Meissner) mientras que los conductores perfectos reaccionan sólo a variaciones de flujo magnético (Ley de Faraday-Lenz). La parte sobre cambios de estado hace la comparación entre diagramas de estado de un sistema sólido-líquido-gaseoso con el diagrama de estado de un superconductor que tiene dos estados: normal y superconductor. Eso se hace de forma cualitativa con el propósito de ayudar a los estudiantes a relacionar un contenido ya conocido (cambio de estado) con un tema nuevo y comparar la organización molecu- 
lar del agua en cada estado con el mayor grado de organización electrónica que ocurre en el paso del estado normal al superconductor. La levitación también se trata de manera cualitativa a través del uso de dibujos y pequeños párrafos explicativos.

De manera análoga, la teoría BCS es presentada con el uso de un dibujo que muestra la formación de pares de Copper a partir de la atracción de dos electrones por la distorsión de la red cristalina. Algunas analogías se utilizan en el texto: el «efecto colchón» (que compara la red cristalina con un colchón y los dos electrones con dos esferas pesadas y cargadas negativamente) y el «efecto dominó» (una analogía para la ausencia de resistividad en un superconductor por el movimiento ordenado de pares de Cooper). Superconductores de tipo II son presentados a través de su diagrama de estado y tres dibujos ilustran los tres estados de ese tipo de superconductor. Nuevamente, muchos dibujos y pequeños párrafos fueron utilizados. Al final, algunos ejemplos de aplicaciones tecnológicas de la superconductividad fueron presentadas en las ultimas cinco hojas del texto.

Cada futuro enseñante dictó un promedio de diez clases de 50 minutos a los alumnos de secundaria. El tópico de partículas fue enfocado desde una perspectiva más «informativa» mientras que el de superconductividad tuvo un enfoque más «explicativo». Sin embargo, en los dos casos se intentó facilitar un aprendizaje significativo receptivo.

El proceso de enseñanza-aprendizaje de temas modernos se organizó en la perspectiva de la teoría del aprendizaje significativo receptivo en muchos de sus aspectos, tanto desde el punto de vista del futuro profesor como desde el del alumno de secundaria. Mapas conceptuales fueron utilizados para facilitar la comprensión por parte de los futuros profesores sobre partículas elementales (leptones y quarks; bosones mediadores; hadrones -mesones y bariones) e interacciones fundamentales (gravitacional, electromagnética, fuerte y débil), así como inspiraron la elaboración de una tabla en formato póster sobre ese tópico. El concepto de aprendizaje receptivo como una «modalidad» importante de aprendizaje significativo fue siempre nuestro marco teórico. Creemos que incluso en un área como la física de partículas en la que no hay experimentos para hacer en el aula, alumnos de nivel medio pueden entender significativamente muchos de sus conceptos por aprendizaje receptivo. Obviamente, los subsunsores relevantes deben ser identificados y el alumno debe tenerlos. Por ejemplo, como se argumentó anteriormente, una relación interesante para ese tópico son los modelos atómicos discutidos en aulas de química. Para la «superconductividad», también identificamos los subsunsores necesarios (en la física clásica): cambio de estado (de la termodinámica) y ley de Faraday-Lenz (del electromagnetismo) son algunos ejemplos. Los conceptos de diferenciación progresiva y reconciliación integradora también fueron muy explorados. Por ejemplo, a través de las interacciones ya conocidas (gravitacional y electromagnetica), el concepto de interacción fue reconciliado integrativamente juntando también las interacciones (nucleares) fuerte y débil. Además, el propio concepto (más general) se reformuló en la perspectiva de la física moderna: un cuerpo interactúa con otro vía intercambio de una partícula mediadora, y todas las interacciones pueden ser así descritas.

El aprendizaje de los alumnos fue evaluado a través de pretests y postests de conocimiento, a los cuales fueron agregados algunos ítems diseñados para tener una idea de su receptividad con respecto a esos nuevos tópicos (uno de esos instrumentos está en el anexo).

El desempeño de los estudiantes-docentes fue evaluado a través de observaciones de sus clases, en un informe y una entrevista final con la profesora de «Práctica de Enseñanza». Las observaciones de la mayoría de las clases de los futuros docentes fue simplemente el uso de «diarios de a bordo», sin la utilización de audio o vídeo. Ese trabajo de observación consiste en hacer anotaciones de todo lo que parece relevante. Después de cada clase, se acostumbra a hacer comentarios sobre las observaciones hechas a cada docente-estudiante, en el sentido de acompañamiento que permite mejoras a lo largo del proceso. Se procura también no hacer interferencias en el momento del aula, para que el futuro profesor tenga su autoridad garantizada delante de la clase. Los informes finales elaborados son trabajos bastante completos, incluyendo una descripción de todas las etapas de la «Práctica de Enseñanza» y su análisis. Al final del semestre, cada futuro profesor fue entrevistado por la profesora de «Práctica». Las entrevistas fueron todas grabadas y tuvieron un promedio de duración de 15 minutos. Básicamente, se hicieron las siguientes preguntas, a cada docente-estudiante:

- ¿Qué valor atribuyes a la introducción de la física moderna y contemporánea en la enseñanza media?

- ¿Cuáles son las dificultades encontradas para enseñar tópicos contemporáneos? Ventajas y desventajas. ¿Valió la pena? ¿Por qué?

- Como profesor de escuela, ¿repetirías esta propuesta de introducción de tópicos contemporáneos en la enseñanza media?

- ¿Qué importancia tuvo para su formación esa experiencia en la «Práctica de Enseñanza»?

- En relación con la estructura de la asignatura, ¿pareció buena? ¿Hay que cambiar algo?

Las entrevistas fueron analizadas y, junto con las observaciones de las clases y con los informes finales, constituyeron los datos cualitativos de esa investigación, cuya síntesis se presenta a continuación.

\section{RESULTADOS}

Desde el punto de vista del futuro profesor, la experiencia de enseñar tópicos de física contemporánea en secun- 
daria como parte de las tareas de la asignatura «Práctica de Enseñanza de la Física» puede, a partir del análisis que hicimos de los informes finales y de las entrevistas individuales, ser sintetizada como sigue.

- Todos los futuros docentes han atribuido mucha importancia a la problemática de la actualización curricular en física. En general, consideraron que es necesario repensar el currículo de física en secundaria con el objetivo de incluir tópicos más nuevos en él.

- Respecto a las dificultades enfrentadas en las escuelas, las más mencionadas fueron: indisciplina de los alumnos, falta de prerequisitos, mucha abstracción requerida en algunos aspectos de los tópicos, inseguridad respecto al dominio del contenido.

- Los 12 estudiantes-profesores afirmaron, con convicción, la intención de incorporar tópicos actuales de física en su práctica docente futura. A todos les pareció que los tópicos de partículas y superconductividad habían despertado el interés de los alumnos. Sin embargo, han sugerido también otros tópicos.

- En general, consideraron que la asignatura «Práctica de Enseñanza» estuvo bien organizada y ha contribuido a su formación no sólo en lo que se refiere a la enseñanza sino también en términos de contenidos de física, pues, a pesar de estar en el último semestre de su curso, tenían fallos conceptuales en los contenidos que iban a enseñar.

- Otro aspecto positivo destacado por los futuros enseñantes fue el hecho de que ellos mismos elaboraron el texto del alumno en vez de recibirlo listo para usar. No obstante, dijeron que el texto del profesor les ayudó mucho para aprender el contenido y preparar el texto y otros materiales para los alumnos.

- El examen de ingreso a la universidad fue mencionado, con frecuencia, como un obstáculo en la actualización curricular en física, dado que el programa del examen no incluye tópicos contemporáneos y, por ende, el profesor no puede «perder tiempo» con esos tópicos.

Desde la perspectiva del aprendizaje de los alumnos de secundaria y de su receptividad a temas nuevos en física, los resultados de los pretests y postests sugieren, en general, que han aprendido, en la medida que se pueda inferir aprendizaje a través de esos tests, algunos conceptos de superconductividad o de partículas elementales y, además, que se motivaron y tuvieron despierta su curiosidad respecto a la física de hoy a pesar de que no estaría en el examen de ingreso a la universidad.

Por ejemplo, los datos numéricos indican que en los «grupos de partículas», al final, el $43 \%$ de los alumnos ha aprendido el modelo atómico actual (contra el $0 \%$ al comienzo) y el $57 \%$ ha percibido que son sólo cuatro las interacciones fundamentales de la naturaleza. En esos mismos grupos, el $47 \%$ de los estudiantes ha manifestado interés en aprender más sobre el tópico de partículas.

Estos datos están en la tabla I, en la que se presenta el porcentaje de respuestas a la primera pregunta de los cuestionarios inicial y final del «grupo de las partículas». En la ultima columna, aparece el nivel de significancia estadística obtenido con el test $t$. Ese nivel indica la probabilidad de que las diferencias encontradas pueden ser atribuidas a fluctuaciones estadísticas, o sea, que no sean estadísticamente significativas.

Tabla I

Porcentaje de respuestas dadas a la pregunta 1: ¿Cuál es la imagen que tienes de un átomo? Intenta dibujarla en una figura.

\begin{tabular}{|lccc|}
\hline Categorías & $\begin{array}{c}\text { Porcentaje } \\
\text { de respuestas } \\
\text { iniciales }\end{array}$ & $\begin{array}{c}\text { Porcentaje } \\
\text { de respuestas } \\
\text { finales }\end{array}$ & $\begin{array}{c}\text { Nivel } \\
\text { de significancia } \\
\text { estadística }\end{array}$ \\
\hline 1. Electrones, neutrones y protones & $13,0 \%$ & $22,0 \%$ & 0,07 \\
\hline 2. Bola pequeña sin partes & $13,0 \%$ & $1,0 \%$ & 0,00 \\
\hline 3. Círculos concéntricos sin indicaciones & $25,0 \%$ & $9,0 \%$ & 0,00 \\
\hline 4. Núcleo y electrones & $33,0 \%$ & $15,0 \%$ & 0,57 \\
\hline 5. Pudín de pasas & $0,8 \%$ & $1,6 \%$ & 0,00 \\
\hline 6. Orbitales son indicados & $38,0 \%$ & $15,0 \%$ & 0,00 \\
\hline 7. Modelo actual & $0,0 \%$ & $43,0 \%$ & 0,57 \\
\hline 8. No ha aprendido nada / No sabe nada & $2,4 \%$ & $1,6 \%$ & 0,48 \\
\hline 9. No contestó a la pregunta & $2,4 \%$ & $4,0 \%$ & 0,00 \\
\hline 10. Modelo actual incompleto & $0,0 \%$ & $13,0 \%$ & 0,04 \\
\hline 11. Átomo indivisible & $4,8 \%$ & $1,6 \%$ & 0,08 \\
\hline 12. Estados de la materia & $2,4 \%$ & $0,0 \%$ & \\
\hline
\end{tabular}


Tabla II

Porcentaje de respuestas dadas a la pregunta 3: ¿Cuáles son los tipos de fuerzas que conoces? Da ejemplos de fuerzas que observas en la naturaleza o que ya hayas estudiado. ¿Es posible clasificarlas? ¿Son muchas?

\begin{tabular}{|c|c|c|c|}
\hline Categorías & $\begin{array}{c}\text { Porcentaje } \\
\text { de respuestas } \\
\text { iniciales }\end{array}$ & $\begin{array}{c}\text { Porcentaje } \\
\text { de respuestas } \\
\text { finales }\end{array}$ & $\begin{array}{c}\text { Nivel } \\
\text { de significancia } \\
\text { estadística }\end{array}$ \\
\hline 1. Lista de fuerzas estudiadas u observadas & $90,0 \%$ & $48,0 \%$ & 0,00 \\
\hline 2. Confusión con otros conceptos físicos & $13,0 \%$ & $3,0 \%$ & 0,01 \\
\hline 3. Dos fuerzas: gravitacional y electromagnética & $0,8 \%$ & $5,6 \%$ & 0,03 \\
\hline $\begin{array}{l}\text { 4. Cuatro fuerzas: gravitacional, } \\
\text { electromagnética, fuerte y débil }\end{array}$ & $0,0 \%$ & $57,0 \%$ & 0,00 \\
\hline 5. No ha aprendido nada / No sabe nada & $0,0 \%$ & $12,0 \%$ & 0,00 \\
\hline 6. No contestó a la cuestión & $4,8 \%$ & $4,0 \%$ & 0,76 \\
\hline 7. Es posible clasificarlas pero no sabe cómo & $2,4 \%$ & $0,8 \%$ & 0,32 \\
\hline 8. Tres fuerzas & $0,0 \%$ & $7,0 \%$ & 0,00 \\
\hline
\end{tabular}

La tabla II muestra el porcentaje de respuestas para la tercera pregunta en los cuestionarios inicial y final del «grupo de las partículas».

En la tabla II, se puede constatar que, inicialmente, el $90 \%$ de los alumnos creía que había una infinidad de tipos de fuerzas. Por otro lado, al final, el 57\% percibió la simplicidad de la naturaleza a partir de sus cuatro interacciones fundamentales.

En los «grupos de superconductividad» también se detectaron, numéricamente, evidencias de aprendizaje. Por ejemplo, casi el $40 \%$ de los alumnos relacionó, al final, la resistividad nula con el efecto Meissner y los pares de Cooper. En ese grupo, el 58\% de los alumnos ha dicho que le gustaría aprender más sobre el tópico.

La tabla III muestra el porcentaje de respuestas para la pregunta 2 del cuestionario final para el «grupo de la superconductividad».

Tabla III

Porcentaje de respuestas para la pregunta 2 del cuestionario final ¿Te gustaría saber más sobre este tema? ¿La superconductividad despertó en ti alguna curiosidad especial?

$\begin{array}{lr}\text { Categorías } & \text { Porcentaje de } \\ & \\ \text { 1. No me gustaría saber más } & 26 \% \\ \text { 2. Me gustaría saber más } & 58 \% \\ \text { 3. No despertó curiosidad } & 14 \% \\ \text { 4. Despertó curiosidad } & 48 \% \\ \text { 5. No contestó a la pregunta } & 14 \%\end{array}$

Puede verse, por la tabla III, que el fenómeno de la supercondutividad no sólo despertó curiosidad en el $48 \%$ de los alumnos, sino que también a un $58 \%$ le gustaría saber más sobre el mismo. Sin embargo, nuestro objetivo no es tanto el presentar números en este trabajo. Hicimos toda la comparación estadística entre el pretests y postest y las diferencias fueron estadísticamente significativas. Además, la opinión unánime de los futuros profesores es que los resultados de aprendizaje de sus alumnos fueron, según su percepción, plenamente satisfactorios. No obstante, lo que nos parece más importante en este momento es compartir y discutir nuestra experiencia.

\section{DISCUSIÓN Y CONCLUSIÓN}

La primera etapa de nuestra experiencia fue la realización del estudio Delphi a través del cual llegamos a una lista de tópicos modernos y contemporáneos que, según físicos, investigadores en enseñanza de la física y profesores de física, podrían o deberían ser enseñados en secundaria si quisiéramos actualizar el currículo de física en ese nivel. Nos llamó la atención en ese estudio el hecho de que los expertos no establecen una clara diferencia entre tópicos modernos y contemporáneos. Sin embargo, la mezcla de tópicos modernos y contemporáneos en la lista final, aunque pueda estar señalando que dicha distinción no es importante, seguramente sugiere que el currículo de física en secundaria debe ser urgentemente actualizado pues, a lo mejor, lo que se enseña de física hoy en las escuelas (no sólo de Brasil, creemos) es todavía premoderno. En la etapa de producción de recursos didácticos hemos constatado que es necesario invertir en la producción de materiales instruccionales sobre temas de física contemporánea (y moderna) si se quiere 
actualizar el currículo de física en las escuelas, tanto para profesores como para alumnos. Esos temas en general no están contemplados en los materiales disponibles para profesores y alumnos. Sin embargo, no es tarea trivial, pues escribir un texto o preparar un software, por ejemplo, sobre un tema de física contemporánea (y moderna) requiere conocimiento y una cierta madurez en física así como en enseñanza-aprendizaje y diseño instruccional.

En la fase de preparación de los profesores nos convencemos de que sí es una fase crucial del proceso de actualización curricular. De nada sirve cambiar los programas con el fin de incluir tópicos contemporáneos toda vez que son los profesores quienes van a implementar esos tópicos en el aula y ellos pueden no estar preparados en términos de contenidos o no estar convencidos del cambio. Es decir, la actualización curricular en física pasa necesariamente por la actualización de los profesores en cuanto al contenido o por una predisposición para el cambio.

En nuestro caso encontramos una salida que nos resultó muy buena. Trabajamos con futuros docentes muy jóvenes, muy abiertos a cosas nuevas y con la física muy fresca en sus cabezas. No fue difícil prepararlos en los nuevos contenidos, a pesar de que tenían huecos conceptuales, ni convencerlos de que valía la pena enseñar dichos contenidos en secundaria. Les faltaba la experiencia docente, el manejo de clase, pero eso lo han superado en buena medida con el desarrollo de su práctica docente. Obviamente, sería poco realista imaginar que fuera posible una mejora acentuada en el manejo de las actividades en el aula durante un período de tiempo relativamente corto. Sin embargo, fue posible percibir progresos a lo largo de ese período, pues hicimos un acompañamiento casi permanente de sus clases, en el cual al final de cada una de esas sesiones, el docente recibía un feedback respecto a su trabajo docente.

No obstante, nos parece que normalmente la situación no será así de favorable al cambio curricular. La resistencia de los profesores, por varias y legítimas razones, es, seguramente, un factor que hay que tener en cuenta en el proceso de actualización curricular en física o, más explícitamente, en la incorporación de tópicos de física contemporánea (y moderna) en el currículo de física de las escuelas.
Finalmente, en la última etapa, en la bajada al aula, observamos y quedamos convencidos de que los alumnos de secundaria pueden aprender, significativamente, tópicos de física contemporánea. Es un error pensar que ellos no tienen capacidad (de abstracción, por ejemplo) para aprender esos tópicos. La cuestión es cómo enfocarlos en la enseñanza. En nuestra experiencia utilizamos recursos instruccionales especialmente preparados, analogías y vinculación con tópicos clásicos ya existentes en el currículo.

Constatamos también que los estudiantes, a pesar ser de aparentemente indisciplinados (quizás tenían sólo la natural agitación típica de la edad), se sintieron más motivados para el estudio de la física. Nótese que usualmente los alumnos tienen una actitud un poco negativa con respecto a los «aprendices de profesor», pero eso no ha pasado (de forma muy acentuada) en nuestra experiencia, quizás por lo interesante de los temas.

Naturalmente, la inclusión de temas nuevos y motivadores en el currículo de física en secundaria no debe implicar un aumento en la extensión de ese currículo. Hay que sacar temas, hay que cambiar objetivos. Los alumnos pueden interesarse mucho más por temas como el origen del universo, la estructura de la materia, la relatividad, el láser, los semiconductores y los superconductores que en la aburrida cinemática, en la abstracta segunda ley de Newton o en cómo funcionan algunas cosas de su mundo cotidiano.

En resumen, nos parece que hay que actualizar el currículo de física en secundaria a través de la incorporación de tópicos modernos y contemporáneos y de la exclusión de tópicos clásicos. No obstante, para eso es necesario preparar materiales y profesores. Los alumnos parece que ya están listos.

\section{NOTA}

${ }^{1}$ A lo largo de las tres etapas hubo participantes que no contestaron los cuestionarios, como suele ocurrir en este tipo de técnicas. Sin embargo, al final nos quedamos con 76 que respondieron los tres cuestionarios. Este número fue considerado satisfactorio, dado que en la época del estudio el uso del correo electrónico todavía no estaba ampliamente difundido entre los participantes, en particular los profesores. 


\section{REFERENCIAS BIBLIOGRÁFICAS}

ALEMAÑ BERENGUER, R. A. (1997). Errores comunes sobre relatividad entre los profesores de enseñanza secundaria. Enseñanza de las Ciencias, 15(3), pp. 301-307.

ALLDAY, J. (1997). The nature of force in particle physics. Physics Education, 32(5), pp. 327-332.

ALVETTI, M.A.S. y DELIZOICOV, D. (1998). Ensino de física moderna e contemporânea e a Revista Ciência en Encontro de pesquisa em ensino de física, 6. Florianópolis. Atas. Florianópolis: Imprensa UFSC, 3 p., (Seção de Pôsteres). 1 CD-ROM.

ARONS, A.B. (1990). A guide to introductory physics teaching. Nueva York: John Wiley.

AUBRECHT, G.J. (1989). Redesigning courses and textbooks for the twenty-first century. American Journal of Physics, 57(4), pp. 352-359.

AUBRECHT, G.J. (1986). Report on the conference on the teaching of modern physics. The Physics Teacher, 24(9), pp. 540-546.

AUSUBEL, D.P. (1963). The psychology of meaningful verbal learning. Nueva York: Grune \& Stratton.

AUSUBEL, D.P. (1968). Educational psychology: a cognitive view. Nueva York: Hott, Rinehart and Winston.

AUSUBEL, D.P., NOVAK, J.D. y HANESIAN, H. (1983). Psicología educativa: un punto de vista cognoscitivo. México: Trillas.

BARLOW, R. (1992). Particle physics: from school to university. Physics Education, 27(2), pp. 92-95.

BAROJAS, J. (ed.) (1988). Cooperative networks in physics education. Nueva York: American Institute of Physics (AIP Conference Proceedings, 173).

CAMARGO, A.J. (1996). A introdução de física moderna no $2^{\circ}$ grau: obstáculos e possibilidades. Florianópolis: Curso de Pós-Graduação em Educação - UFSC, Diss. mestr. Educação.

CLOSE, F. (1983). The cosmic onion: quarks and the nature of the universe. Londres: Heinemann Educational Books.

CONTEMPORARY PHYSICS EDUCATION PROJECT (1998). Internet: http://www-pdg.lbl.gov/cpep.html. $23 \mathrm{dez}$.

CUPPARI, A., RINAUDO, G., ROBUTTI, O. y VIOLINO, P. (1997). Gradual introduction of some aspects of quantum mechanics in a high school curriculum. Physics Education, 32(5), pp. 302-308.

CYROT, M. y PAVUNA, D. (1992). Introduction to superconductivity and high-T $T_{c}$ materials. Singapur: World Scientific.

DE POSADA APARICIO, J.M. y PRIETO RUZ, T. (1990). Exploraciones gráficas de ideas extraescolares de los alumnos sobre radiactividad. Enseñanza de las Ciencias, 8(2), pp. 127-130.

DUIT, R. (1993). Research on student's conceptions-developments and trends, en International Seminar on Misconceptions and Educational Strategies in Science and Mathematics, 3. Ithaca. Atas. Ithaca: Cornell University, 1993. 32p. (Oral Communications).

FARMELO, G. (1992). Teaching particle physics in the open university's science foundation course. Physics Education, 27(2), pp. 96-101.
FERMILAB (1998). Discovering the nature of nature. Internet: http://www.fnal.gov./Fermilab. 23 dez.

FISCHLER, H. y LICHTFELDT, M. (1992). Modern physics and students' conceptions. International Journal of Science Education, 14(2), pp. 181-190.

FURIÓ, M.C.J. (1994) Tendencias actuales en la formación del profesorado de ciencias. Enseñanza de las Ciencias, 12(2), pp. 188-199.

GALETTI, D. (1990). Fusão nuclear com múons. Caderno Catarinense de Ensino de física, 7(3), pp. 203-211.

GIL, D.P., SENENT, F. y SOLBES, J. (1987). La introducción a la física moderna: un ejemplo paradigmático de cambio conceptual. Enseñanza de las Ciencias, núm. extra, pp. 209210.

GIL, D.P., SENENT, F. y SOLBES, J. (1988) Análisis critico de la introducción de la física moderna en la enseñanza media. Revista de Enseñanza de la Física, 2(1), pp. 16-21.

GIL, D.P. y SOLBES, J. (1993). The introduction of modern physics: overcoming a deformed vision of science.International Journal of Science Education, 15(3), pp. 255-260. Londres.

IRESON, G. (1996). Relativity at A-level: a looking glass approach. Physics Education, 31(65), pp. 356-361.

JONES, D.G.C. (1992). Cosmology and particle physics. Physics Education, 27(2), pp. 76-80.

KALMUS, P. I. (1992). Particle physics at A-level-the universities' viewpoint. Physics Education, 27(2), pp. 62-64.

LIJNSE, P.L.,EIJKELHOF, H.M.C., KLAASSEN, C.W.J.M. y SCHOLTE, R.L.J. (1990). Pupils' and mass-media ideas about radioactivity. International Journal of Science Education, 12(1), pp. 67-78.

LINSTONE, A . H. y TUROFF, M. (1975). The Delphi Method: tecniques and applications. Massachusetts: AddisonWesley.

LUIZ, A.M. (1992). Aplicações de supercondutividade. São Paulo: Edgard Blücher.

MOREIRA, M.A. (1990). Um mapa conceitual para interações fundamentais. Enseñanza de las Ciencias, 8(2), pp. 133-139.

NOBEL, J. DE. (1996). The discovery of superconductivity. Physics Today, 49(9), pp. 40-42.

NOVAK, J.D. y GOWIN, D.B. (1988). Aprendiendo a aprender. Barcelona: Martínez Roca.

OSTERMANN, F. (1998). Um texto para professores do ensino médio sobre partículas elementares. (Aceptado para publicación en la Revista Brasileira de Ensino de física.)

OSTERMANN, F., FERREIRA, L.M. y CAVALCANTI, C.J.H. (1998a). Tópicos de física contemporânea no ensino médio: um texto para professores sobre supercondutividade. Revista Brasileira de Ensino de física, 20(3), pp. 270-288.

OSTERMANN, F., FERREIRA, L.M. y CAVALCANTI, C.J.H. (1998b). Supercondutividade: uma proposta de inserção no ensino médio. Porto Alegre: Instituto de Física. UFRGS.

OSTERMANN, F. y MOREIRA, M.A. (1998). Tópicos de física contemporânea na escola média brasileira: um estudo com a técnica Delphi, en Encontro de pesquisa em ensino de física, 6. Florianópolis.Atas. Florianópolis: Imprensa UFSC, 19 p. (Seção de Comunicações Orais) 1 CD-Rom. 


\section{INVESTIGACIÓN DIDÁCTICA}

PARTICLE PHYSICS GROUP (1997). Particle physics. Londres: Particle Physics and Astronomy Research Council.

PEREIRA, O. da S. (1997). Raios cósmicos: introduzindo física moderna no $2^{o}$ grau. São Paulo: Instituto de física e Faculdade de Educação. USP, Diss. mestr. Ensino de Ciências.

PORLÁN, R. y RIVERO, A. (1998). El conocimiento de los profesores: una propuesta en el área de ciencias. Sevilla: Díada.

PUREUR NETO, P. (1996). Supercondutividade. Porto Alegre: Curso de Pós-Graduação em física. UFRGS. Notas de aula.

ROSE-INNES, A.C. y RHODERICK, E.H. (1988). Introduction to superconductivity. Oxford: Pergamon.

SCHWARZ, C. (1992). A tour of the subatomic zoo: a guide to particle physics. Nueva York: American Institute of Physics.

SOLBES, J., CALATAYUD, M.L., CLIMENT, J.B. y NAVARRO, J. (1987a). Diseño de un currículo para la introducción del modelo atómico cuántico. Enseñanza de las Ciencias, 5, núm. extra, pp. 209-210.

SOLBES, J., CALATAYUD, M.L., CLIMENT, J.B. y NAVARRO, J. (1987b). Errores conceptuales en los modelos atómicos cuánticos. Enseñanza de las Ciencias, 5(3), pp. 189-195.

STANNARD, R. (1990). Modern physics for the young. Physics Education, 25(3), pp. 133.

STEFANEL, A. (1998). Una experiencia en el marco de la introducción de la física cuántica en la escuela secundaria. Revista de Enseñanza de la Física, 11(2), pp. 35-44.

SWINBANK, E. (1992). Particle Physics: a new course for schools and colleges. Physics Education, 27(2), pp. 87-91.

TERRAZZAN, E.A. (1992). A inserção da física moderna e contemporânea no ensino de física na escola de $2^{\circ}$ grau. Caderno Catarinense de Ensino de física, 9(3), pp. 209-214.

TERRAZZAN, E.A. (1994). Perspectivas para a inserção de física moderna na escola média. Tesis doctoral. São Paulo: Curso de Pós-Graduação em Educação. USP.

WILLIAMS, W.S.C. (1992). Nuclear and particle physics. Nueva York: Oxford University Press.

WILSON, B. (1992). Particle physics at A-level-a theacher's viewpoint. Physics Education, 27(2), pp. 64-65 (Bristol).

[Artículo recibido en junio de 1999 y aceptado en noviembre de 1999.] 
ANEXO

CUESTIONARIO SOBRE PARTÍCULAS ELEMENTALES

\section{Cuestionario inicial}

1) ¿Cuál es la imagen que tienes de un átomo? Intenta dibujarla en una figura.

2) ¿Tienes idea de cuál(les) es(son) la (s) más pequeña (s) porción (nes) que forman toda la materia en el universo? Explícalo con tus palabras.

3) ¿Cuáles son los tipos de fuerzas que conoces? Da ejemplos de fuerzas que observes en la naturaleza o que ya hayas estudiado. ¿Es posible clasificarlas? ¿Son muchas?

Cuestionario final: Las tres preguntas iniciales más una cuarta pregunta y preguntas objetivas.

4) ¿Te gustaría saber más sobre física de partículas? ¿Ese tema despertó en ti alguna curiosidad especial?

\section{ELIGE LA MEJOR RESPUESTA EN CADA ÍTEM}

1) Un pedazo de hierro y una cierta cantidad de agua tienen en común:

a) los átomos

b) las moléculas

c) protones, neutrones y electrones

2) El átomo es:

a) indivisible

b) divisible en dos partes

c) divisible en más de dos partes

3) Los protones y los neutrones:

a) están constituidos por porciones más pequeñas

b) no están constituidos por porciones más pequeñas

c) unos están constituidos por porciones más pequeñas y otros no

4) El número de tipos de fuerzas que conoces o de las cuales ya escuchaste hablar es:

a) más de cien

b) entre diez y cien

c) menos de diez

5) En el núcleo, los protones se repelen mutuamente, ¿cómo, entonces, permanecen juntos en un mismo núcleo?

a) debido a la presencia de los neutrones

b) debido a la presencia de los electrones

c) porque hay otro tipo de fuerza que los mantiene unidos

6) Los electrones, según el modelo actual de la estructura de la materia:

a) están constituidos por porciones más pequeñas

b) no están constituidos por porciones más pequeñas

c) no se sabe si están o no constituidos por porciones más pequeñas

7) La fuerza gravitacional es:

a) siempre repulsiva

b) siempre atractiva

c) a veces, atractiva y, a veces, repulsiva

8) La fuerza electromagnética es:

a) a veces, atractiva y, a veces, repulsiva

b) siempre atractiva

c) siempre repulsiva

9) La fuerza fuerte residual:

a) atrae neutrinos y protones

b) atrae electrones y protones

c) atrae protones y protones 


\section{INVESTIGACIÓN DIDÁCTICA}

10) La fuerza fuerte fundamental:

a) es responsable de la formación de los átomos

b) actúa en partículas con carga de color

c) actúa sobre leptones

11) La fuerza débil:

a) es responsable de la estabilidad del núcleo

b) es la fuerza responsable del decaimiento beta

c) es la fuerza de menor intensidad en la naturaleza

12) Relaciona la partícula mediadora (columna de la izquierda) con la fuerza (columna de la derecha):

a) gluón débil

b) gravitón fuerte

c) $\mathrm{W}, \mathrm{Z} \quad$ electromagnética

d) fotón gravitacional

13) El antiprotón tiene:
a) carga positiva
b) carga negativa
c) no tiene carga

14) El neutrón y el antineutrón tienen:

a) la misma masa

b) pequeña o ninguna masa

c) la misma constitución de quarks

15) El pión:

a) tiene masa menor que la del protón

b) es un leptón

c) no tiene estructura interna

16) Es un leptón:

a) el electrón

b) el neutrón

c) el protón

17) Son hadrones:
a) los quarks
b) los neutrinos
c) los mesones

18) Los quarks:
a) tienen estructura interna
b) forman los hadrones
c) forman los leptones

19) Los mesones están formados por:
a) tres quarks
b) tres antiquarks
c) un quark y un antiquark

20) Los bariones están formados por:
a) tres quarks
b) dos quarks
c) un quark y un antiquark

21) Es un ejemplo de mesón y de barión, respectivamente:
a) pión y neutrón
b) protón y quark
c) electrón y neutrino 